\title{
O CONHECIMENTO VEICULADO EM LITERATURA NACIONAL SOBRE INTEGRALIDADE EM SAÚDE*
}

Taysa Sant’Ana Ferreira1, Luciana de Freitas Campos²

RESUMO: Este estudo teve por objetivos levantar o que tem sido publicado na forma de artigos em periódicos nacionais no período de 10 anos e destacar os principais aspectos abordados sobre integralidade em saúde. Foi realizada revisão de literatura em 10 periódicos de saúde, online, no período de 1997 a 2007, sendo selecionados 15 artigos que versavam sobre o tema. Integralidade vem se construindo na saúde coletiva, no ensino e na atenção hospitalar, no entanto, é contextualizada de maneira diversa em cada um destes campos a partir de diferentes concepções veiculadas em literatura nacional. Verificou-se que o tema vem sendo debatido entre profissionais e que há esforços para a amplitude de reflexão e consolidação de conceito e princípios, o que requer mudanças na maneira de organização do trabalho vigente e também nas relações humanas.

PALAVRAS-CHAVE: Assistência integral à saúde; Educação; Sistema único de saúde.

\section{THE KNOWLEDGE TRANSMITTED IN NATIONAL LITERATURE ABOUT INTEGRALITY IN HEALTH}

\begin{abstract}
This study aims to survey the national literature on papers regarding "integrality in health" during the last 10 years and highlight the main aspects approached on this subject. Literature review was performed, in 10 health journals, on line, from 1997 to 2007, and 15 articles were selected The theme integrality is being developed in public health, in education and hospital care, however it's contextualized differently in each of these fields from different conceptions reported in national literature. It appears that the issue is being discussed among professionals and that there are efforts to extend the reflections and consolidation of the concept and its principles. This requires changes in the work organization and also in human relations.
\end{abstract}

KEYWORDS: Comprehensive health care; Education; Single health system.

\section{EL CONOCIMIENTO VEHÍCULADO EN LA LITERATURA NACIONAL SOBRE INTEGRIDAD EN LA SALUD}

RESUMEN: Este estudio tuvo como objetivos levantar lo que ha sido publicado en forma de artículos en periódicos nacionales en el periodo de 10 años y destacar los principales aspectos abordados sobre la integridad en salud. Fue realizada revisión de la literatura, en 10 periódicos de salud, online, durante el período de 1997 a 2007, siendo seleccionados 15 artículos que versaban sobre el tema. Integridad viene construyéndose en la salud colectiva, en la educación y en la atención hospitalaria, entretanto, es contextualizada de manera diversa en cada uno de estos campos a partir de diferentes concepciones vehiculadas en la literatura nacional. Se verificó que el tema está siendo debatido entre profesionales y que hay esfuerzos para la amplitud de reflexión y consolidación de conceptos y principios, lo que requiere cambios en la forma de organización del trabajo vigente y también en las relaciones humanas.

PALABRAS CLAVE: Atención integral a la salud; Educación; Sistema unico de salud.

\footnotetext{
*Artigo extraído do Trabalho de Conclusão de Curso apresentado ao Departamento de Enfermagem da Universidade Federal dos Vales do Jequitinhonha e Mucuri-UFVJM, vinculado ao Grupo de Estudos em Gerenciamento dos Serviços de Saúde e Enfermagem, cadastrado no CNPq.

${ }^{1}$ Discente do Curso de Graduação em Enfermagem da UFVJM. Diamantina-MG.

${ }^{2}$ Enfermeira. Especialista em Enfermagem do Trabalho. Mestre em Enfermagem Fundamental. Docente da UFVJM.
}

Autor correspondente:

Luciana de Freitas Campos

Rua da Glória, 187 - 39100-000 - Diamantina-MG

Recebido: 28/11/08

E-mail: camposlf@gmail.com 


\section{INTRODUÇÃO}

No decorrer do século XX diversas transformações ocorreram na sociedade brasileira no âmbito econômico, cultural, político, social e também no tecnológico, exigindo mudanças na atenção à saúde por meio da revisão de avanços nas políticas de saúde. Em especial, a partir da década de 70, impulsionado pelo Movimento da Reforma Sanitária, que culminou na criação do Sistema Único de Saúde-SUS, determinando as diretrizes para a saúde da população, garantidas na Constituição da República Federativa do Brasil, promulgada em 1988. Nesta, o principal avanço para a sociedade consistiu no entendimento de que a saúde é direito de todos os cidadãos brasileiros e dever do Estado, sendo regido pelos princípios da universalidade, participação popular, municipalização, equidade e integralidade.

A integralidade origina-se do grego hole ousia, apontando para o sentido ser saudável e completo, a referência para a saúde ${ }^{(1)}$. Como princípio do SUS, é garantida ao usuário a atenção que abrange ações de promoção, prevenção, tratamento e reabilitação da saúde, com garantia de acesso a todos os níveis de complexidade. Pressupõe a assistência focada no indivíduo, na família e na comunidade. É entendida também como conjunto de ações livre do reducionismo (fragmentação do cidadão frequentemente adotada pelos profissionais de saúde), em que a assistência é caracterizada por tratar do sujeito imbuído de sentimentos, desejos, preocupações. Portanto, o atendimento integral vai além do tratamento da doença e o cuidado deve ser considerado nas suas dimensões técnica, política, social, econômica, cultural e espiritual.

Nela, o modo de entender e abordar o indivíduo baseia-se na teoria holística, na qual o homem não pode ser explicado apenas por seus aspectos físico, psicológico e social, ou seja, avaliados separadamente ${ }^{(2)}$. Assim, o cuidado deve ir além do modelo biomédico e conduzir ao cuidado da mente, corpo e alma ${ }^{(3)}$, orientada para a escuta e compreensão ampliada do indivíduo com vistas $\mathrm{a}$ atender às demandas e necessidades, segundo a dinâmica do processo saúde-doença.

Diante do exposto, concorda-se que ela só pode ser obtida em rede, ou seja, os serviços devem ser organizados na lógica do cuidar, objetivando organizar o fluxo do indivíduo no serviço de saúde de acordo com sua necessidade, baseado na "linha do cuidado" cada qual operando distintas tecnologias ${ }^{(4)}$. Nessa perspectiva, a trajetória da saúde do usuário, tanto na atenção básica quanto hospitalar, é de conhecimento dos profissionais de saúde, favorecendo a tomada de decisões para o cuidado ao indivíduo com continuidade.

Logo, é no contexto dos serviços de saúde que a integralidade se desenvolve e o seu cumprimento contribui para a qualidade da atenção e melhoria na qualidade de vida do indivíduo, garantindo assim o acesso do mesmo aos diversos níveis de complexidade. Este talvez seja o princípio menos visível na trajetória desse "novo" sistema e suas práticas, pois as mudanças não têm sido tão evidentes, apesar de estar sendo um assunto almejado.

A assistência à saúde requer de seus profissionais habilidades tanto técnicas quanto de relacionamento interpessoal e tem acontecido embasada pelo modelo biomédico com enfoque curativo. Uma nova lógica vem sendo discutida e extrapola a real atenção individual e coletiva, voltada para o olhar ao ser humano como um todo, o que pressupõe a valorização da prática do cuidar em saúde enquanto política governamental e princípio do SUS.

Durante a graduação em enfermagem na Universidade Federal dos Vales do Jequitinhonha e Mucuri houve uma aproximação teórica com o tema integralidade. Entretanto, a articulação entre a teoria e a prática apresentava déficit e o modo como os serviços de saúde e o currículo estão organizados acaba sendo um dificultador, devido à fragmentação do cuidado, alto grau de especialização, divisão de tarefas, característica do modelo hegemônico, que tende a perpetuar um modelo individualista de assistência. Nos estágios supervisionados, tanto na atenção básica quanto hospitalar, foi possível uma aproximação diferenciada com o cotidiano de trabalho em saúde, por meio da assistência direta e indireta bem como dos trabalhos acadêmicos solicitados. Assim, surgiu a inquietação referente a como implementar a integralidade no atendimento em saúde, uma vez que essa prática não era evidenciada no cotidiano dos serviços vivenciados, entendendo que se faz necessária e importante para a prestação do cuidado holístico ao indivíduo.

Assim, este estudo teve por objetivos levantar o que tem sido produzido na literatura nacional nos últimos 10 anos em artigos acerca da integralidade e destacar os principais aspectos abordados sobre o tema.

\section{METODOLOGIA}

Realizou-se um estudo exploratório-descritivo de revisão de literatura publicada em periódicos de 
ciências da saúde no período de 1997 a 2007. Para tal foram selecionadas 10 revistas devido sua viabilidade de acesso, em idioma da língua portuguesa, por meio da consulta ao site da CAPES. São elas: Revista Gaúcha de Enfermagem, Revista Ciência \& Saúde Coletiva, Revista Mineira de Enfermagem, Revista Eletrônica de Enfermagem, Revista Cogitare Enfermagem, Revista Latino Americana de Enfermagem, Revista da Escola de Enfermagem da Universidade de São Paulo, Revista de Saúde Pública, Cadernos de Saúde Pública, Revista de Enfermagem da Universidade do Estado do Rio de Janeiro, Interface - comunicação, saúde, educação. Os descritores utilizados foram: saúde integral, assistência integral à saúde, atenção à saúde, Sistema Único de Saúde, humanização, integralidade.

Foram consultados os sites das revistas selecionadas, considerando nesta busca inicial o título e resumo dos artigos. A seguir, foram selecionados aqueles na íntegra, via online. Realizou-se, então, a leitura exploratória efetuando-se uma seleção para determinação do material de interesse para a investigação, delimitando-se 14 artigos que abordaram a integralidade.

De posse do material coletado procedemos à leitura exaustiva do mesmo, estabelecendo uma relação aberta com o artigo, permitindo que se revelasse em suas intenções, num diálogo investigador-texto.

\section{RESULTADOS E DISCUSSÃO}

A seguir ressaltamos algumas idéias que emergiram da análise dos artigos, de modo que foram destacados os principais aspectos abordados sobre o tema integralidade em saúde.

Dentro do período delimitado para a revisão de literatura, o primeiro artigo encontrado com exposição do assunto foi de 1998, a produção bibliográfica sobre a temática tem mais destaque a partir de 2000.

Após a Constituição de 1988 a saúde, antes almejada por muitos e com acesso para poucos, passou a ser um direito de todos e dever do Estado. Entre as ações promulgadas neste ato está a integralidade do cuidado, voltada para a prevenção e promoção da saúde, sem deixar de ser assistencial.

\section{CONCEPÇÕES E PRINCÍPIOS DE INTEGRALIDADE}

A integralidade da assistência é uma diretriz do
SUS, vem sendo almejada e discutida principalmente na última década, porém não há ainda uma definição precisa em toda sua totalidade. Em alguns textos é definida como ações de promoção, prevenção, reabilitação e tratamento prestados a um indivíduo e sua família: “[...] prevê-se nesse conceito que sejam ofertadas ações de promoção de saúde, prevenção de fatores de risco, assistência aos danos e reabilitação [...]”(5:573). É uma ação livre do reducionismo, da fragmentação do sujeito que deve ser visto de forma holística, compreendido em todas suas necessidades, sejam físicas, espirituais, sociais e/ou psicológicas

\begin{abstract}
a integralidade [...] pode ser percebida como um conjunto de ações livre do reducionismo; com uma visão abrangente do ser humano, tratando não somente como seres doentes, mas como pessoas dotadas de sentimentos, desejos e aflições ${ }^{(2: 43)}$.
\end{abstract}

Ainda, a integralidade do cuidado pode ser vista em diferentes dimensões: voltada ao serviço de saúde bem como ao profissional; este busca compreender as necessidades do serviço de saúde, relacionado à política de acordo com as respostas dadas pelo governo aos problemas existentes na saúde a fim de melhorála e referente a organização das práticas ${ }^{(2,6,7)}$.

Caracteriza-se pela percepção ampliada das necessidades e também das habilidades em reconhecer a oferta de ações de acordo com o contexto de cada situação, no encontro do sujeito com o profissional de saúde ${ }^{(7)}$.

O diálogo é visto como premissa para se alcançar a integralidade, pois através da escuta, da conversa, da troca de informação, é possível conhecer o sujeito como um todo. Seus medos, expectativa em relação à doença, conhecimento acerca da patologia e, a partir de então, poder contribuir para sua melhora. Deste modo, contribui para que o sujeito entenda e se envolva efetivamente no seu projeto terapêutico ${ }^{(1-2,7-9)}$ : "a partir do diálogo e intercâmbio de saberes técnico-científicos e populares, profissionais e usuários podem contribuir de forma compartilhada um saber sobre o processo saúdedoença" $(9: 48)$.

Além disso, o vínculo e o acolhimento são apontados também como uma maneira para se alcançar a integralidade. Existem relações de maior proximidade entre o profissional e o sujeito, nas quais o primeiro consegue ajudar mais o usuário por estabelecer estreitamento de laços, de confiança ${ }^{(2,7,10-13)}:$ “[...] a construção do vínculo nas relações entre profissional e usuário é um desafio para a integralidade do 
cuidado"(12:55). Ainda, há relato acerca da atenção dada ao sujeito ao ouvir, de forma qualificada, a garantia de acesso a todas as pessoas, dar resolubilidade, além de ser uma diretriz para as políticas de saúde. Toda pessoa que procura atendimento em um serviço de saúde deve ter reposta concreta desse serviço, sendo a escuta atenta uma das propostas chave do acolhimento ${ }^{(7)}$.

\section{A INTEGRALIDADE E CAMPOS DE ATUAÇÃO}

Ter integralidade é também ter acesso a todos os níveis de complexidade, ter acesso à tecnologia necessária naquele dado momento, independente de qual. A tecnologia deve relacionar-se ao cuidado propriamente dito, ao vínculo, à escuta, ao acolhimento, ao saber científico, às clínicas médicas, aos equipamentos e exames modernos que trabalham com precisão $^{(2,13)}$

os níveis de complexidade estão dispostos em três [...] o nível primário está qualificado para atender e resolver os principais problemas que demandam serviços de saúde [...], o nível secundário são os centros de especialidades, e no nível terciário de atenção à saúde estão os hospitais de referência(2:43).

Apesar de o avanço tecnológico ter trazido mais rapidez e precisão a exames e equipamentos e cura para algumas doenças, por outro lado levou ao afastamento entre o profissional de saúde e o usuário, o que vem interferir no alcance da integralidade.

É percebida uma dissociação para se conceituar a integralidade no âmbito da saúde pública em relação ao ambiente hospitalar. Na primeira, é tida como uma assistência voltada para a prevenção e promoção da saúde, não deixando de ser reabilitadora, implicando uma variedade de ações

de acordo com o princípio da integralidade o Programa de Saúde da Família deve ofertar prioritariamente assistência promocional, preventiva, sem, contudo descuidar da atenção curativa e reabilitadora ${ }^{(9: 49)}$.

No segundo, é vista como uma prestação de serviço desenvolvida por uma equipe multiprofissional, voltada para a assistência, dependente da disponibilidade de equipamentos para acesso à tecnologia necessária. Sua falta é percebida como uma dificuldade ao atendimento integral: "o cuidado [...] no hospital [...] depende da conjugação do trabalho de vários profissionais. [...] a maior ou menor integralidade da atenção recebida resulta [...] da forma como se articula a prática [...]”(14).

Para alguns autores ${ }^{(4,13-14)}$ trabalhar a integralidade a partir da linha do cuidado, permite ações que integram uma visão global das condições de vida e objetiva organizar o fluxo de acordo com as necessidades do indivíduo. Assim, evita a fragmentação do ser, contribuindo para o alcance dos princípios dos SUS e, consequente, satisfação do sujeito cuidado de forma mais acolhedora, dialógica e empática.

Um dos desafios para se alcançar a integralidade do cuidado é reorganizar a forma como vários estabelecimentos trabalham até os dias de hoje, devendo haver mudança na prática dos profissionais e também na maneira como o serviço é organizado $(2,5,8-9)$. O modelo assistencial vigente precisa melhor organizar os serviços nos diversos níveis de atenção à saúde para que o acesso seja garantido assim como o cuidado integral. Outro desafio está voltado para os profissionais de saúde que necessitam incorporar, na prática, a solidariedade e reconhecer o sujeito do cuidado e da atenção à saúde como autônomo ${ }^{(1)}$.

Alguns autores ${ }^{(8,13)}$ atribuem o desafio da integralidade ao fato das práticas estarem voltadas para procedimentos e não para o cuidado.

A compreensão da integralidade está relacionada ao modo de perceber o sujeito no contexto dos serviços de saúde, do acesso ao mesmo a este serviço, das ações ofertadas e a maneira na qual é ofertada. Neste contexto, para se obter a integralidade é necessário que haja mudança na relação entre profissional-objeto para entre profissional-sujeito, baseado no diálogo, na troca de informação: "é indispensável que o profissional tenha mais sensibilidade, escute o outro, saiba o que ele pensa, numa postura que não seja distante e impessoal” (5:581).

A garantia do princípio da integralidade, em suma, implica dotar o sistema de condições relacionado às diversas fases da atenção à saúde, ao processo de cuidar, ao relacionamento do profissional de saúde com os pacientes $^{(5: 577)}$.

A territorialização, por sua vez, é vista como uma forma de alcançar a integralidade pelo fato do trabalho ocupar uma área delimitada, dificultando, assim, definir as prioridades locais e interferir de modo a promover a saúde, melhorar a qualidade de vida do sujeito e sua família, desenvolver o acolhimento e o vínculo. "É preciso compreender que melhorar a qualidade de vida, promover a saúde de um indivíduo, implica agir no 
contexto em que ele se insere $[\ldots . . .]^{\prime(5: 578)}$.

Ressaltou-se a importância do sistema de informação para obter a integralidade, pois contribui para a sua construção na medida em que as informações obtidas podem ser transformadas em melhoria para a saúde ${ }^{(15)}$.

A atenção básica tem sido vista como uma alternativa para alcançar a integralidade e por isso priorizada $^{(5,8-11)}$. Também pelo fato de se ter uma equipe de saúde com profissionais mais próxima da população e por priorizar a prevenção e promoção à saúde.

\section{A INTEGRALIDADE E O ENSINO}

A integralidade, a partir da análise de alguns $\operatorname{artigos}^{(2,12)}$, vem emergindo como temática a ser enfatizada no contexto da saúde/enfermagem/ensino. O ensino fragmentado e o desinteresse em se manter atualizado são dificuldades encontradas para alcançar a integralidade.

Construir a integralidade do cuidado em saúde implica assumir o agir em saúde como princípio educativo em uma nova forma de apreender/ensinar em saúde, que rompe com o saber formatado e descontextualizado ${ }^{(12: 55)}$.

A integralidade é abordada no processo de formação profissional ${ }^{(4,8-9,12)}$ no contexto do ensino na graduação. Foi apontada a valorização da importância de mudanças na grade curricular, pois os conhecimentos têm sido construídos de forma fragmentada; o sujeito é percebido em partes. É necessário desde a graduação um trabalho em equipe, ambiente de aprendizagem variado, formação críticoreflexiva do profissional, visão global do mundo, indivíduos conhecedores dos princípios do SUS e que visem à formação de um profissional mais interessado pelos resultados.

No âmbito da educação algumas escolas ainda refletem e reproduzem uma lógica fragmentada e deixam de lado as relações interpessoais. Entende-se que há a necessidade de mudanças nesta lógica e articulação entre academia e serviços de saúde, formando o contexto pelo qual a integralidade possa se desenvolver tanto na atenção básica quanto na atenção hospitalar.

\section{CONSIDERAÇÕES FINAIS}

A integralidade na assistência à saúde vem sendo discutida na atenção básica, atenção hospitalar e no ensino em saúde. Este movimento permite-nos inferir que os profissionais têm debatido a questão procurando desenvolver estratégias para implementála. Entretanto, na prática, tanto na assistência quanto no ensino há necessidade de se somarem esforços para que a integralidade na assistência à saúde seja operacionalizada e efetivada.

A integralidade requer mudanças na maneira em que a organização do trabalho se dá no modelo de gestão predominante e nas relações humanas no trabalho.Com o desenvolvimento deste estudo espera-se contribuir para ampliar as discussões sobre o tema com perspectivas a minimizar o déficit na articulação entre a teoria e a prática da integralidade em saúde.

\section{REFERÊNCIAS}

1. Costa AM. Integralidade na atenção e no cuidado à saúde. Saúde Soc. 2004;13(3):5-15.

2. Pinho IC, Siqueira JCBA, Pinho, LMO. As percepções do enfermeiro acerca da integralidade da assistência. Rev. Eletron. Enferm. 2006;8(1):42-51.

3. Fonseca ALN, Lacerda MR, Maftum MA. O cuidado transpessoal de enfermagem no domicílio ao portador de transtorno mental e sua família. Cogitare Enferm. 2006 Jan/Abr;11(1):7-15.

4. Feuerwerker LCM, Cecílio LCO. O hospital e a formação em saúde: desafios atuais. Cienc Saúde Col. 2007;12(4): 965-71.

5. Campos CEA. O desafio da Integralidade segundo as perspectivas da vigilância da saúde e da saúde da família. Cienc Saúde Col. 2003;8(2):569-84.

6. Hartz ZMA, Contandriopoulos AP. Integralidade da atenção e integração de serviços de saúde: desafios para avaliar a implantação de um "sistema sem muros". Cad Saúde Publ. 2004;20(supl.2):S331-S6.

7. Mattos RA. A integralidade na prática (ou sobre a prática da integralidade). Cad Saúde Publ. 2004;20(5):1411-6.

8. Machado MFAS, Monteiro EMLM, Queiroz DT, Vieira NFC, Barroso MGT. Integralidade, formação de saúde, educação em saúde e as propostas do SUS: uma revisão conceitual. Cienc Saúde Col. 2007;12(2):335-42.

9. Alves VS. Um modelo de educação em saúde para o Programa Saúde da Família: pela integralidade da atenção e reorientação do modelo assistencial. Interface, Saúde e Comunicação. 2005;9(16):39-52. 
10. Gomes MCPA, Pinheiro R. Acolhimento e vínculo: práticas de integralidade na gestão do cuidado em saúde em grandes centros urbanos. Interface, Saúde e Comunicação. 2005;9(17):287-301.

11. Pinheiro R. Atenção básica à saúde: um olhar a partir das práticas de integralidade em saúde. Rev Min Enferm. 2005;9(2):174-8.

12. Silva KL, Sena RR. Integralidade do cuidado na saúde: indicações a partir da formação do enfermeiro. Rev Esc Enferm USP. 2008;42(1):48-56.

13. Malta DC, Cecílio LCO, Merhy EE, Franco TB, Jorge AO, Costa MA. Perspectivas da regulação na saúde suplementar diante dos modelos assistenciais. Cienc Saúde Col. 2004;9(2):433-44.

14. Cecílio LCO, Merhy EE. Integralidade do cuidado como eixo da gestão hospitalar. In: Pinheiro R, Mattos R. Construção da integralidade: cotidiano, saberes e práticas em saúde. Rio de Janeiro: IMS/Abrasco; 2003.

15. Veras MMS, Quinderé PHD, Ferreira LP, Aragão JMG, Coelho MAAA. Sistema de informação dos núcleos de atenção integral na saúde da família-SINAI. Saúde Soc. 2007;16(1):165-71. 